УДК 541.138.3

\title{
ВЛИЯНИЕ АНОДИРОВАНИЯ НА КИНЕТИКУ ВЫДЕЛЕНИЯ ВОДОРОДА НА СИЛИЦИДАХ КОБАЛЬТА В РАСТВОРЕ СЕРНОЙ КИСЛОТЫ
}

\author{
() 2017 В. И. Кичигин, А. Б. Шеин \\ Пермский государственный нацииональный исследовательский университет, \\ ул. Букирева, 15, 614990 Пермь, Россия \\ e-mail: ashein@psu.ru \\ Поступила в редакцию 29.05.2017 г.
}

\begin{abstract}
Аннотация. Изучено влияние анодирования $\mathrm{Co}_{2} \mathrm{Si}$ - и $\mathrm{CoSi}_{2}$-электродов в $0.5 \mathrm{M} \mathrm{H}_{2} \mathrm{SO}_{4}$ при потенциалах формирования оксида $E_{f}$ от 0.4 до 2.0 В (с.в.э.) на кинетику реакции выделения водорода (РВВ) в $0.5 \mathrm{M} \mathrm{H}_{2} \mathrm{SO}_{4}$. Установлено, что поведение анодного оксида на силицидах кобальта с низким и высоким содержанием Si существенно различается: оксидные пленки на $\mathrm{Co}_{2} \mathrm{Si}$, полученные при всех изученных $E_{f}$, катодно восстанавливаются; оксидные пленки на $\mathrm{CoSi}_{2}$ сохраняются в катодной области. Сделан вывод, что на анодированном CoSi $\mathrm{C}_{2}$-электроде PBB протекает на границе оксид/раствор с участием туннелирующих через оксидную пленку электронов; лимитирующей стадией является разряд ионов водорода.
\end{abstract}

Ключевые слова: силицид кобальта, анодирование, реакция выделения водорода, туннелирование электронов.

\section{ВВЕДЕНИЕ}

Кинетика и механизм реакции выделения водорода (РВВ) на силицидах переходных металлов исследованы в ряде работ [1-8]. Некоторые результаты (например, высокие значения тафелевского наклона $b \approx 0.2 \mathrm{~B}$ на FeSi-электроде [4]) могут быть объяснены влиянием тонкого оксидного слоя на поверхности электрода. Такое влияние предполагалось в $[8,9]$ для силицидов железа и кобальта в растворе $\mathrm{H}_{2} \mathrm{SO}_{4}$ при невысоких катодных поляризациях.

Основу оксидов на многих силицидах составляет $\mathrm{SiO}_{2}$ [10]. Имеются данные о влиянии плотных тонких пленок $\mathrm{SiO}_{2}$ (1.5-10 нм), напыленных на Pt-электрод, на кинетику PBВ в растворе серной кислоты [11]. Показано, что с увеличением толщины пленки диоксида кремния катодный ток при данном потенциале электрода уменьшается, однако пленки обеспечивают перенос заряда, достаточный для протекания РВВ. В качестве вероятных причин заметной проводимости тонких пленок $\mathrm{SiO}_{2}$ называются квантовое туннелирование, образование ионов $\mathrm{OH}^{-}$на оксидном слое или наличие дефектов на границах зерен оксида [11].

Чтобы сделать ситуацию с влиянием поверхностных оксидов на силицидах металлов более яс- ной, проведено исследование РВВ на электродах из силицидов кобальта $\mathrm{Co}_{2} \mathrm{Si}$ и $\mathrm{CoSi}_{2}$, на которых предварительно сформированы оксидные пленки путем потенциостатической поляризации при различных потенциалах электрода (потенциалах формирования $E_{f}$ оксида) в пассивной и транспассивной областях в растворе серной кислоты.

\section{МЕТОДИКА ЭКСПЕРИМЕНТА}

Исследуемые электроды изготовлены из силицидов кобальта $\mathrm{Co}_{2} \mathrm{Si}_{\text {и }} \mathrm{CoSi}_{2}$, полученных методом Чохральского из кремния КПЗ-1 (99.99 мас.\% Si) и электролитического кобальта К-0 (99.98 мас.\% Со). Рабочая площадь поверхности электродов составляла 0.4-0.5 см². Поверхность электродов обрабатывали на тонкой шлифовальной бумаге, очищали этиловым спиртом и промывали рабочим раствором, а именно $0.5 \mathrm{M} \mathrm{H}_{2} \mathrm{SO}_{4}$.

Растворы готовили из кислоты марки «х.ч.» и деионизованной воды (Milli-Q, удельное сопротивление 18.2 МОм·см, содержание органического углерода 3-4 мкг/л). Температура растворов составляла $21-23{ }^{\circ} \mathrm{C}$.

Формирование оксида проводилось в интервале $E_{f}$ от 0.4 до 2.0 В (здесь и далее - относительно стандартного водородного электрода) в течение 


\section{В. И. КИЧИГИН, А. Б. ШЕИН}

1 ч в $0.5 \mathrm{M} \mathrm{H}_{2} \mathrm{SO}_{4}$ в условиях естественной аэрации раствора. Потенциостатические поляризационные кривые, показывающие плотности тока при различных $E_{f}$ на обоих силицидах, приведены на рис. 1. Измерения в катодной области проводили в деаэрированном растворе. Растворы деаэрировали водородом (чистота 99.999 \% в расчете на сухой газ). Потенциал электрода изменяли от более низких катодных поляризаций к более высоким.

Измерения импеданса проводились в диапазоне частот $f$ от 10 кГц (в некоторых случаях - от 100 кГц) до 0.01 Гц в потенциостатическом режиме поляризации с помощью установки Solartron 1255/1287 (Solartron Analytical), амплитуда сигнала составляла 10 мВ. При измерениях и обработке импедансных данных использовали программы CorrWare2, ZPlot2, ZView2 (Scribner Associates, Inc.).

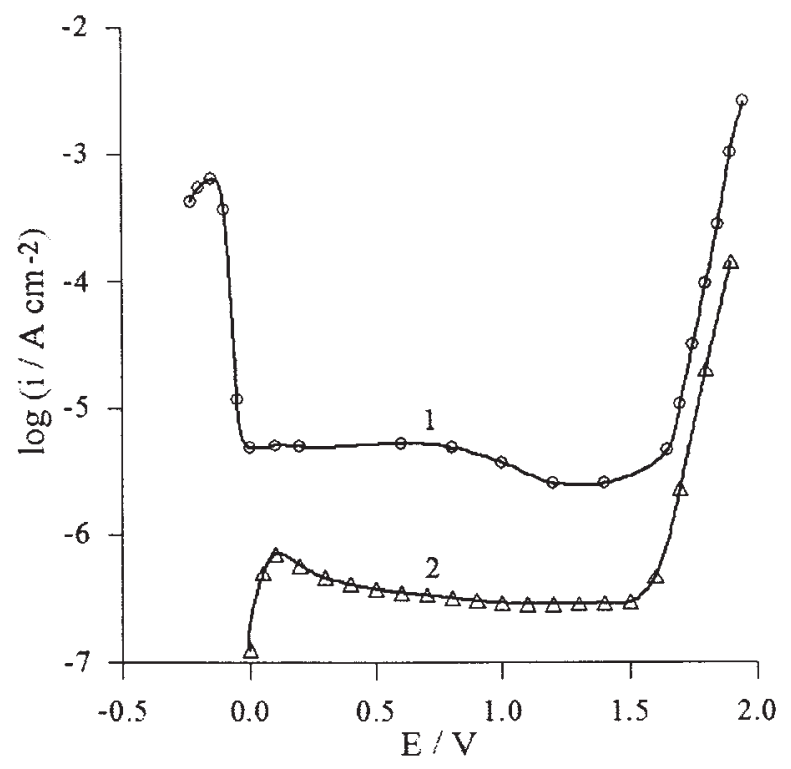

Рис. 1. Анодные поляризационные кривые в $0.5 \mathrm{M} \mathrm{H}_{2} \mathrm{SO}_{4}: 1-\mathrm{Co}_{2} \mathrm{Si} ; 2-\mathrm{CoSi}_{2}$

[Fig. 1. Anodic polarization curves in $0.5 \mathrm{M} \mathrm{H}_{2} \mathrm{SO}_{4}$ : $\left.1-\mathrm{Co}_{2} \mathrm{Si} ; 2-\mathrm{CoSi}_{2}\right]$

\section{РЕЗУЛЬТАТЫ И ИХ ОБСУЖДЕНИЕ}

При анодировании Сo $\mathrm{Si}_{2}$-электрода при $E_{f}=0.4$ В наблюдалась самоактивация электрода после отключения анодной поляризации. При более высоких $E_{f}$ самоактивация не происходила за время порядка 3 ч. Однако при катодной поляризации активация происходит, и катодные поляризационные кривые при различных $E_{f}$ близки друг к другу (рис. 2) и мало отличаются от поляризационной кривой для неанодированного $\mathrm{Co}_{2} \mathrm{Si}$. На электродах, подвергавшихся анодированию, плотности катодного тока даже несколько выше. Это может быть связано с небольшим «растравом» поверхности за время пребывания электрода при анодной поляризации, изменением рельефа поверхности электрода после восстановления оксида, каталитическим эффектом продуктов восстановления оксидной пленки на РВВ. Время катодной активации $t_{a} \mathrm{Co}_{2} \mathrm{Si}$-электрода заметно увеличивается при повышении $E_{f}$ и, например, при $E=-0.24$ В изменяется примерно по закону $\lg t_{a} \approx-0.8+0.7 E_{f}\left(t_{a}-\right.$ в часах, $\left.0.4 \leq E_{f} \leq 2.0 \mathrm{~B}\right)$. За время активации принимали время, отвечающее точке перегиба на зависимости «ток - время» в области быстрого возрастания тока при $E=$ const.

На рис. 3 приведены катодные поляризационные кривые на $\mathrm{CoSi}{ }_{2}$-электроде в $0.5 \mathrm{M} \mathrm{H}_{2} \mathrm{SO}_{4}$. На электроде, не подвергавшемся анодированию, выполняется тафелевская зависимость с наклоном $b$ $=0.093$ В. Сформированный анодный оксид катодно не восстанавливается в $0.5 \mathrm{M} \mathrm{H}_{2} \mathrm{SO}_{4}$ за времена, измеряемые несколькими часами. При всех изученных потенциалах формирования оксида плотности катодного тока меньше плотностей тока на неанодированном $\mathrm{CoSi}_{2}$. При $E_{f}=2.0$ В плотность катодного тока примерно на 2.5 порядка величины меньше по сравнению с неокисленным $\mathrm{CoSi}_{2}$. При

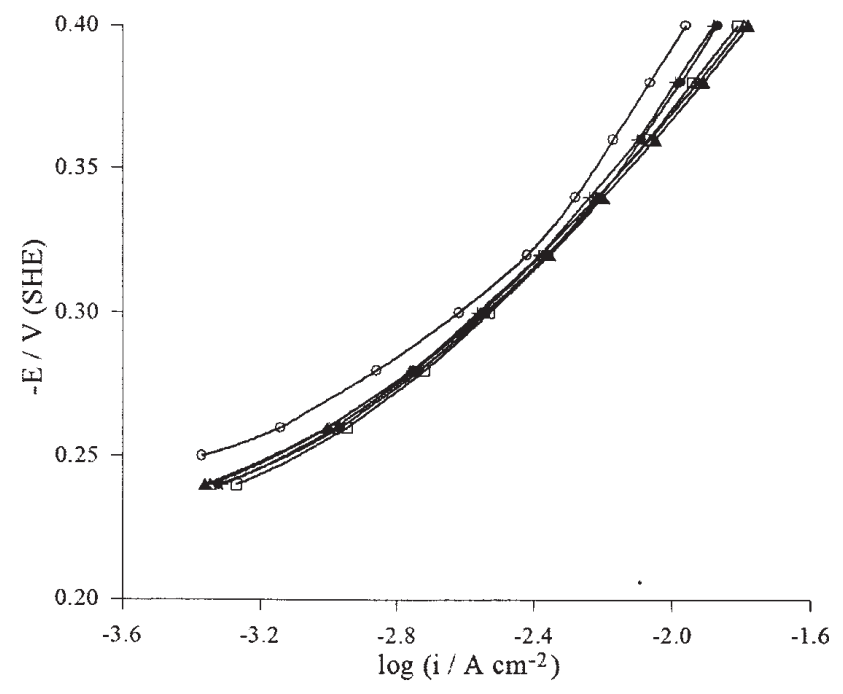

Рис. 2. Катодные поляризационные кривые на $\mathrm{Co}_{2} \mathrm{Si}-$ электроде в $0.5 \mathrm{M} \mathrm{H}_{2} \mathrm{SO}_{4}$ при различных потенциалах формирования оксида $E_{f}, \mathrm{~B}:$ - без анодирования; $\square-$ $0.4 ; \Delta-1.0 ; \boldsymbol{\Delta}-1.5 ;+-1.8 ; \bullet-1.9$

[Fig. 2. Cathodic polarization curves on $\mathrm{Co}_{2} \mathrm{Si}$ in $0.5 \mathrm{M}$ $\mathrm{H}_{2} \mathrm{SO}_{4}$ at different potentials of oxide formation $E_{f}, \mathrm{~V}$ : $\circ-$ without anodising; $\square-0.4 ; \Delta-1.0 ; \boldsymbol{\Delta}-1.5$; + - 1.8; - 1.9 
$E_{f}=0.5-1.5$ В зависимости lgi от $E$ искривлены, их наклон увеличивается при увеличении плотности тока, но при $E_{f}>1.5$ В поляризационные кривые более близки к тафелевским зависимостям. При этом значения $b$ составляют от 0.135 В до $0.165 \mathrm{~B}$, то есть заметно выше, чем на неанодированном электроде. В интервале $E_{f}$ от 1.5 до 1.8 В катодные поляризационные кривые почти сливаются. При $E_{f}=1.9$ и 2.0 В на кривых катодной поляризации в области малых плотностей тока появляется участок очень слабой зависимости тока от потенциала электрода, причем этот предельный ток тем больше, чем выше потенциал формирования оксида (рис. 3).

Быстрое снижение токов РВВ происходит и при потенциалах $E_{f}$ в области перепассивации (рис. 3). Также отметим, что вплоть до $E \approx 4$ В происходит монотонное снижение дифференциальной емкости $\mathrm{CoSi}_{2}$-электрода в $0.5 \mathrm{M} \mathrm{H}_{2} \mathrm{SO}_{4}$ [12]. Эти результаты показывают, что рост оксида в транспассивной области продолжается и барьерные свойства оксидной пленки по отношению к катодной реакции при этом не ослабевают.

Малое влияние катодной поляризации на свойства анодно сформированного оксида на $\mathrm{CoSi}_{2}$-электроде подтверждается результатами емкостных измерений. В табл. 1 приведены значения дифферен-

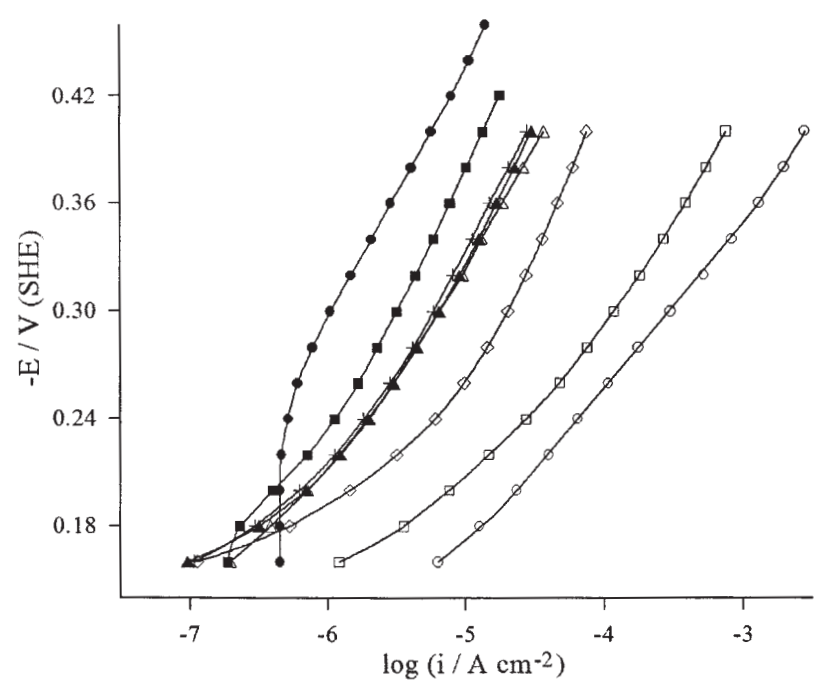

Рис. 3. Катодные поляризационные кривые на $\mathrm{CoSi}_{2}-$ электроде в $0.5 \mathrm{M} \mathrm{H}_{2} \mathrm{SO}_{4}$ при различных потенциалах формирования оксида $E_{f}, \mathrm{~B}:$ - без анодирования; $\square-$ $0.5 ; \diamond-1.0 ; \boldsymbol{\Delta}-1.5 ;+-1.6 ; \triangle-1.7 ; \mathbf{\square}-1.9 ; \bullet-2.0$

[Fig. 3. Cathodic polarization curves on $\mathrm{CoSi}_{2}$ in $0.5 \mathrm{M}$ $\mathrm{H}_{2} \mathrm{SO}_{4}$ at different potentials of oxide formation $E_{p}, \mathrm{~V}: \circ-$ without anodising; $\square-0.5 ; \diamond-1.0 ; \boldsymbol{\Delta}-1.5$; + - 1.6; $\triangle-1.7 ; \mathbf{\square}-1.9 ; \bullet-2.0$ циальной емкости $C$ при частоте 10 кГц при $E=E_{f}$ и $E=-0.16 \mathrm{~B}$; в обоих случаях значения $C$ относятся к времени выдержки 50 мин при указанном потенциале электрода. Видно, что после переключения потенциала из анодной области в катодную и значительной выдержки в катодной области значение $C$ изменяется довольно слабо. Несущественно изменяется емкость и в ходе измерения катодной поляризационной кривой на $\mathrm{CoSi}_{2}$-электроде, окисленном при определенном $E_{f}$ (рис. 4), то есть каждую поляризационную кривую на рис. 3 можно рассматривать как вольтамперную зависимость при постоянной толщине оксидного слоя на электроде.

Таким образом, обнаруживается существенное различие в поведении анодных оксидных слоев на силицидах кобальта с низким и высоким содержанием кремния при катодной поляризации в растворе серной кислоты. На дисилициде кобальта оксид сохраняется в катодной области, в дальнейшем он упрощенно рассматривается как $\mathrm{SiO}_{2}$.

Можно представить два общих механизма РВВ на анодно окисленном $\mathrm{CoSi}_{2}$-электроде в растворе серной кислоты:

1. Образование $\mathrm{H}_{2}$ на границе $\mathrm{CoSi}_{2}$ /оксид. В этом случае будут протекать следующие процессы:

a) адсорбция ионов водорода на поверхности

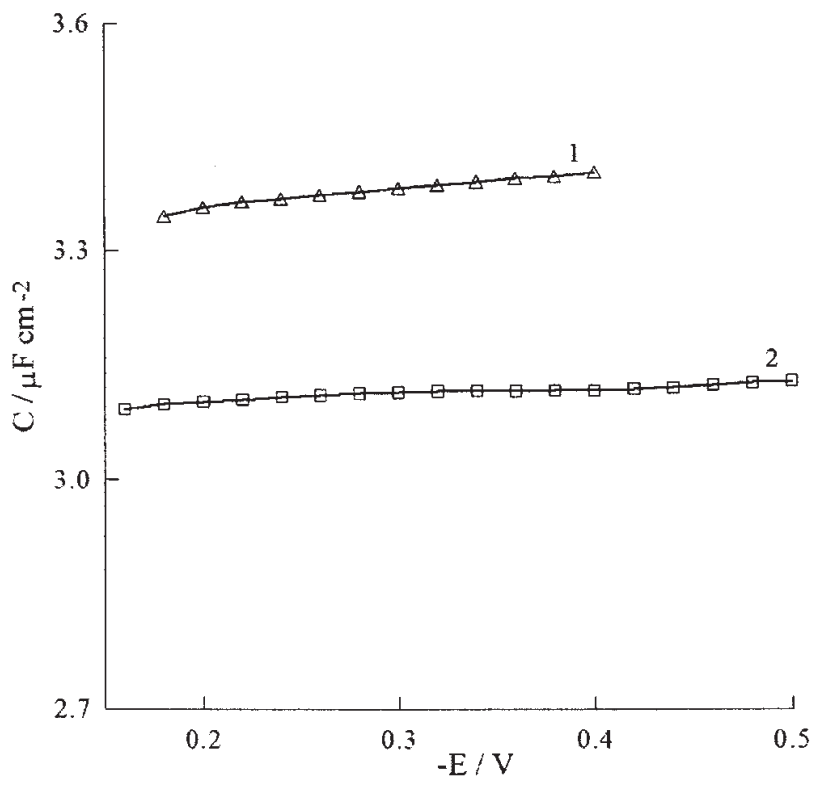

Рис. 4. Кривые дифференциальной емкости, измеренной при частоте 10 кГц, при катодной поляризации анодированных $\mathrm{CoSi}_{2}$-электродов в $0.5 \mathrm{M} \mathrm{H}_{2} \mathrm{SO}_{4}$. Потенциалы формирования оксида, B: $1-1.5 ; 2-2.0$

[Fig. 4. Differential capacitance at $f=10 \mathrm{kHz}$ at cathodic polarization of anodized $\mathrm{CoSi}_{2}$ electrodes in $0.5 \mathrm{M} \mathrm{H}_{2} \mathrm{SO}_{4}$.

Potentials of oxide formation $\left.E_{p}, \mathrm{~V}: 1-1.5 ; 2-2.0\right]$ 
Таблица 1. Значения дифференциальной емкости $\mathrm{CoSi}_{2}$-электрода в $0.5 \mathrm{M} \mathrm{H}_{2} \mathrm{SO}_{4}$ при потенциалах формирования оксида и в катодной области

[Table 1. Differential capacitance of $\mathrm{CoSi}_{2}$ electrode in $0.5 \mathrm{M} \mathrm{H}_{2} \mathrm{SO}_{4}$ at the potentials of oxide formation and in cathodic region]

\begin{tabular}{|c|c|c|}
\hline \multirow{2}{*}{$\begin{array}{l}E_{f}, \mathrm{~B} \\
{\left[E_{p}, \mathrm{~V}\right]}\end{array}$} & \multicolumn{2}{|c|}{ 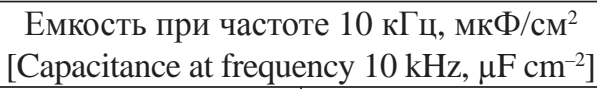 } \\
\hline & $\begin{array}{l}\text { при } E=E_{f} \\
{\left[\text { at } E=E_{f}\right]}\end{array}$ & $\begin{array}{l}\text { при } E=-0.16 \mathrm{~B} \\
\text { [at } E=-0.16 \mathrm{~V} \text { ] }\end{array}$ \\
\hline 0.5 & 4.19 & 4.19 \\
\hline 1.0 & 3.52 & 3.59 \\
\hline 1.5 & 3.20 & 3.33 \\
\hline 1.6 & 3.13 & 3.21 \\
\hline 1.7 & 3.12 & 3.21 \\
\hline 1.8 & 3.08 & 3.24 \\
\hline 1.9 & 2.90 & 3.09 \\
\hline 2.0 & 2.89 & 3.09 \\
\hline
\end{tabular}

оксида - протонирование поверхностных силанольных групп диоксида кремния:

$$
\equiv \mathrm{SiOH}+\mathrm{H}^{+} \rightarrow \equiv \mathrm{SiOH}_{2}^{+} \text {. }
$$

Точка нулевого заряда $\mathrm{SiO}_{2}$ равна 2-3 [13], а значит в $0.5 \mathrm{M} \mathrm{H}_{2} \mathrm{SO}_{4}$ поверхность оксида несет положительный заряд и на ней преобладают группы $\equiv \mathrm{SiOH}_{2}^{+}$;

б) диффузия и миграция $\mathrm{H}^{+}$через оксидный слой к границе раздела $\mathrm{CoSi}_{2} /$ оксид. Протоны в пленке $\mathrm{SiO}_{2}$ могут быстро перемещаться при комнатной температуре под действием электрического поля [14]. Диффузия протонов, вероятно, происходит путем перескоков протона между атомами кислорода [15, 16];

в) разряд протонов с образованием $\mathrm{H}_{\text {адс }}$ на границе $\mathrm{CoSi}_{2}$ /оксид;

г) молизация водорода;

д) диффузия $\mathrm{H}_{2}$ от границы раздела $\mathrm{CoSi}_{2} /$ оксид через оксидную пленку к границе оксид/раствор и далее в объем раствора; процесс диффузии молекулярного водорода в $\mathrm{SiO}_{2}$ описан, например, в [17].

2. Образование $\mathrm{H}_{2}$ на границе оксид/раствор. В этом случае будут протекать следующие процессы:

а) адсорбция ионов водорода на поверхности оксида - реакция (1);

б) туннелирование электронов. При $E_{f}$ до 2 В толщина $d$ анодного оксида на $\operatorname{CoSi}_{2}$ не превышает 2 нм, так как по оценкам [18] при $E>0.2$ В коэффициент анодирования равен 0.36 нм/В. Следует учитывать, что ширина запрещенной зоны аморфного диоксида кремния $E_{g}=9.3$ эВ [19], сродство $\mathrm{SiO}_{2}$ к электрону равно 0.9 эВ [20], а работа выхода дисилицида кобальта равна 4.62-4.77 эВ [21, 22]. Поэтому можно считать, что тонкая пленка $\mathrm{SiO}_{2}$ на поверхности $\mathrm{CoSi}_{2}$-электрода представляет собой высокий, но узкий потенциальный барьер, через который электроны могут туннелировать ;

в) разряд адсорбированного на поверхности оксида протона:

$$
\equiv \mathrm{SiOH}_{2}^{+}+\mathrm{e} \rightarrow \equiv \mathrm{SiOH}(\mathrm{H}) .
$$

г) образование молекулы $\mathrm{H}_{2}$ и ее десорбция:

$$
\equiv \mathrm{SiOH}(\mathrm{H})+\mathrm{H}^{+}+\mathrm{e} \rightarrow \equiv \mathrm{SiOH}+\mathrm{H}_{2} \text {. }
$$

Все перечисленные выше процессы могут протекать на анодированном силициде кобальта. Однако достаточно сильная зависимость кинетики катодной реакции от толщины оксидного слоя свидетельствует в пользу второго варианта.

Важную роль в РВВ по второму механизму играет туннелирование электронов через тонкую пленку оксида. Собственно процесс туннелирования происходит быстро; его влияние на кинетику электродного процесса проявляется через вероятность туннелирования. Известно, что туннелирование Фаулера-Нордгейма характерно для пленок толщиной более 3 нм, причем при достаточно больших напряженностях электрического поля в оксидной пленке [23]. Для рассматриваемого нами случая $(d<2$ нм) типично прямое туннелирование через потенциальный барьер, ширина которого равна толщине оксидного слоя. В зонной структуре дисилицида кобальта отсутствует запрещенная зона [24], то есть $\mathrm{CoSi}_{2}$ ведет себя как металлоподобное соединение. Для прямого туннелирования электронов с уровня Ферми металла (в данном случае - CoSi ${ }_{2}$ ) на незаполненные энергетические уровни редокс-системы в растворе характерна сильная зависимость плотности тока от толщины барьерного слоя на электроде [25]. Для прямоугольного барьера выполняется известное соотношение [26, 27] :

$$
P=\exp \left[-4 \pi d\left(2 m \Delta E_{b}\right)^{1 / 2} / h\right],
$$

где $P$ - вероятность туннелировния, $d-$ ширина потенциального барьера, $m$ - эффективная масса электрона в оксидной пленке, $\Delta E_{b}$ - высота барьера (при нулевом перенапряжении), $h$ - постоянная Планка.

При $E=$ const на анодированном $\mathrm{CoSi}_{2}$-электроде наблюдается примерно экспоненциальное снижение плотности катодного тока при повышении $E_{f}$ до $1.5 \mathrm{~B}$ и при $E_{f} \geq 1.8 \mathrm{~B}$, тогда как в интервале $E_{f}$ от 1.5 до 1.8 В имеется задержка в изменении $\lg i$ (рис. 5). Наклон $d \lg i / d E_{f}$ при $E_{f} \geq 1.8$ В почти в 4 
раза больше, чем при $E_{f} \leq 1.5$ В. Повышение анодной поляризации $\mathrm{CoSi}_{2}$-электрода в растворе $0.5 \mathrm{M}$ $\mathrm{H}_{2} \mathrm{SO}_{4}$ сопровождается пропорциональным увеличением толщины оксидного слоя [12, 18], соответственно рис. 5 можно рассматривать как указание на экспоненциальную зависимость плотности тока от толщины оксидного слоя, согласующуюся с (4).

Отметим, что интервал потенциалов от 1.5 до $1.8 \mathrm{~B}$, в котором имеется задержка в изменении тока на $\lg i, E_{f}-$ зависимости (рис. 5), соответствует началу области транспассивного состояния дисилицида кобальта (рис. 1). В работе [18] область перехода от пассивного состояния к транспассивному ассоциировалась, в соответствии с теорией [28], с увеличением, но лишь до некоторого критического значения, концентрации точечных дефектов в оксидной пленке. При появлении дефектов в тонком оксидном слое механизм туннелирования электронов может измениться: наряду с прямым туннелированием проявляется туннелирование через промежуточные состояния [25] - туннелирование, стимулируемое ловушками, роль которых могут играть точечные дефекты, например кислородные вакансии. Вероятность туннелирования через промежуточные электронные состояния в оксиде выше вероятности прямого туннелирования при отсутствии локальных состояний в запрещенной зоне оксидной пленки той же толщины [25], что

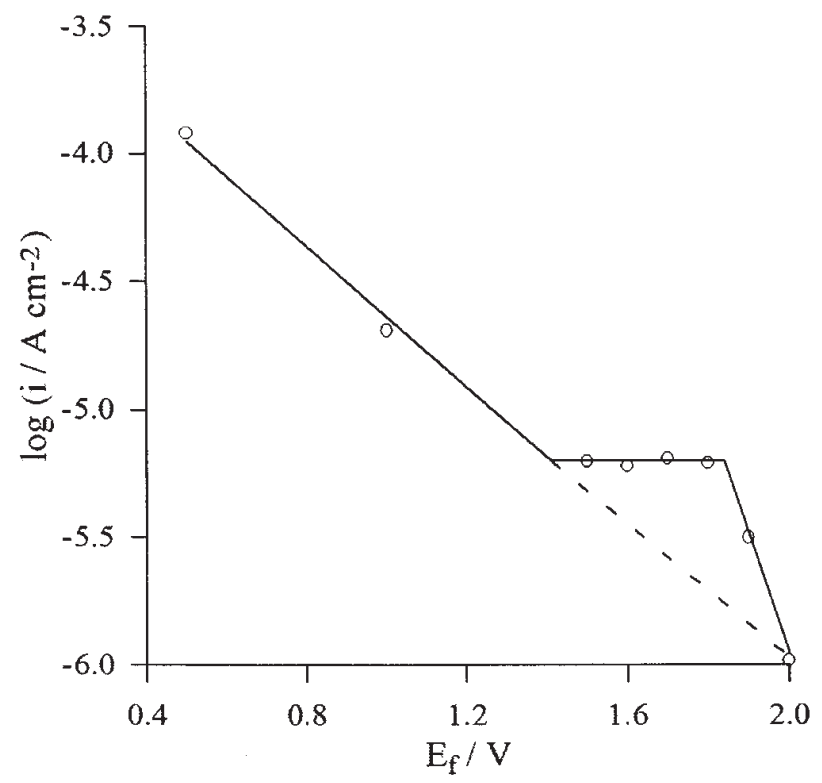

Рис. 5. Зависимость плотности катодного тока на $\mathrm{CoSi}_{2}-$ электроде в $0.5 \mathrm{M} \mathrm{H}_{2} \mathrm{SO}_{4}$ при $E=-0.3$ В от потенциала формирования оксида

[Fig. 5. Dependence of cathodic current density on $\mathrm{CoSi}_{2}$ electrode in $0.5 \mathrm{M} \mathrm{H}_{2} \mathrm{SO}_{4}$ at $E=-0.3 \mathrm{~V}$ on the potential of oxide formation] приводит к увеличению плотности тока. Продолжение прямолинейного участка $\lg i, E_{f}$-зависимости при $E_{f}<1.5$ В к более высоким потенциалам формирования оксида (пунктир на рис. 5) представляет собой зависимость плотности катодного тока от $E_{f}$ при той же электронной структуре оксида, которая существовала при $E_{f}<1.5$ В. Фактическая плотность тока при $E_{f}>1.5$ В превышает экстраполированные значения $i$; максимальное превышение, примерно в 3 раза, достигается при $E_{f} \approx 1.8$ В. При дальнейшем повышении $E_{f}$ до 2.0 В фактические и экстраполированные плотности тока сближаются (рис. 5). По-видимому, накопление дефектов в пленке $\mathrm{SiO}_{2}$ по мере повышения $E_{f}$ вызывает перестройку атомной структуры оксида, что сказывается на барьерных свойствах оксидной пленки и приводит к быстрому спаду плотности катодного тока при $E_{f} \geq 1.8$ В (рис. 5). Структурные изменения в оксидной пленке подготавливают переход в область вторичной пассивации $\mathrm{CoSi}_{2}$, который происходит при $E>2$ В [12].

Проведены измерения импеданса на анодированном $\mathrm{CoSi}_{2}$-электроде в катодной области; примеры графиков импеданса даны на рис. 6. При достаточно низких потенциалах электрода графики Найквиста представляют собой две перекрывающиеся емкостные дуги, то есть имеется некоторое сходс-
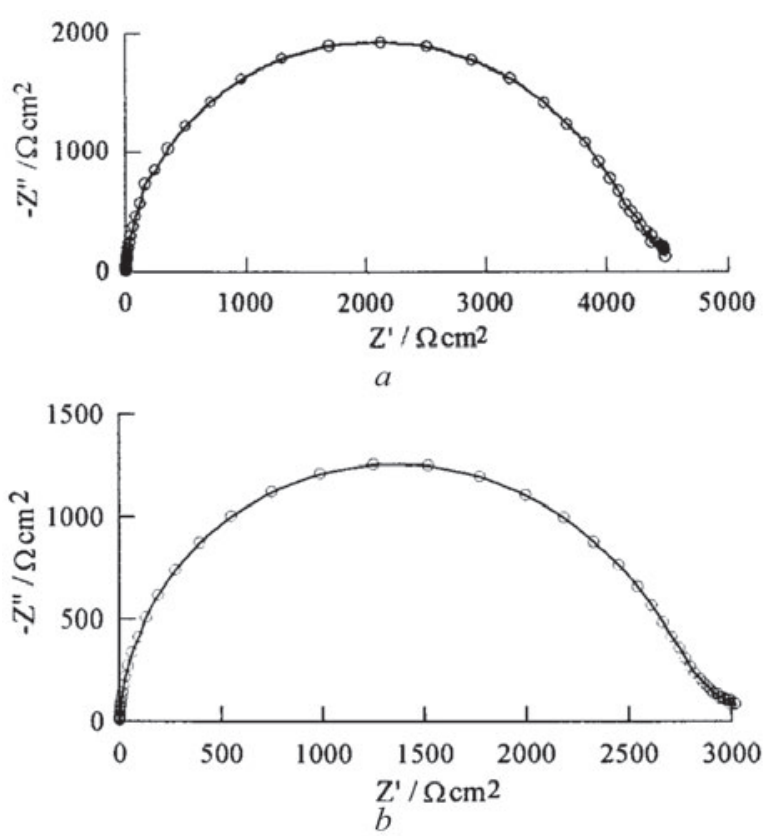

Рис. 6. Графики Найквиста для анодированного $\mathrm{CoSi}_{2}$-электрода в $0.5 \mathrm{M} \mathrm{H}_{2} \mathrm{SO}_{4}: a-E_{f}=1.0 \mathrm{~B}, E=-0.3 \mathrm{~B}$; $b-E_{f}=2.0 \mathrm{~B}, E=-0.5 \mathrm{~B}$

[Fig. 6. Nyquist plots for anodized $\mathrm{CoSi}_{2}$ electrode in $0.5 \mathrm{M}$ $\mathrm{H}_{2} \mathrm{SO}_{4}: a-E_{f}=1.0 \mathrm{~V}, E=-0.3 \mathrm{~V} ; b-E_{f}=2.0 \mathrm{~V}$, $E=-0.5 \mathrm{~V}]$ 


\section{В. И. КИЧИГИН, А. Б. ШЕИН}

тво с графиками импеданса для неанодированного $\mathrm{CoSi}_{2}$-электрода [29]. Однако в случае электрода, не подвергавшегося анодному окислению, перекрывание полуокружностей на графиках импеданса выражено в меньшей степени.

Если РВВ происходит по механизму (2)-(3) или другому двухстадийному механизму, должна выполняться обычная эквивалентная схема (рис. 7). С использованием комплексного нелинейного метода наименьших квадратов (программа ZView) получены значения параметров эквивалентной схемы, которые наилучшим образом описывали экспериментальные графики импеданса при различных катодных потенциалах. На рис. 8 показаны такие зависимости для $\mathrm{CoSi}_{2}$-электрода при $E_{f}=2.0$ В. В полулогарифмических координатах зависимости являются линейными; их наклоны

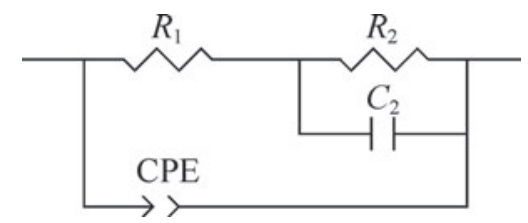

Рис. 7. Эквивалентная электрическая схема

[Fig. 7. Equivalent electrical circuit]

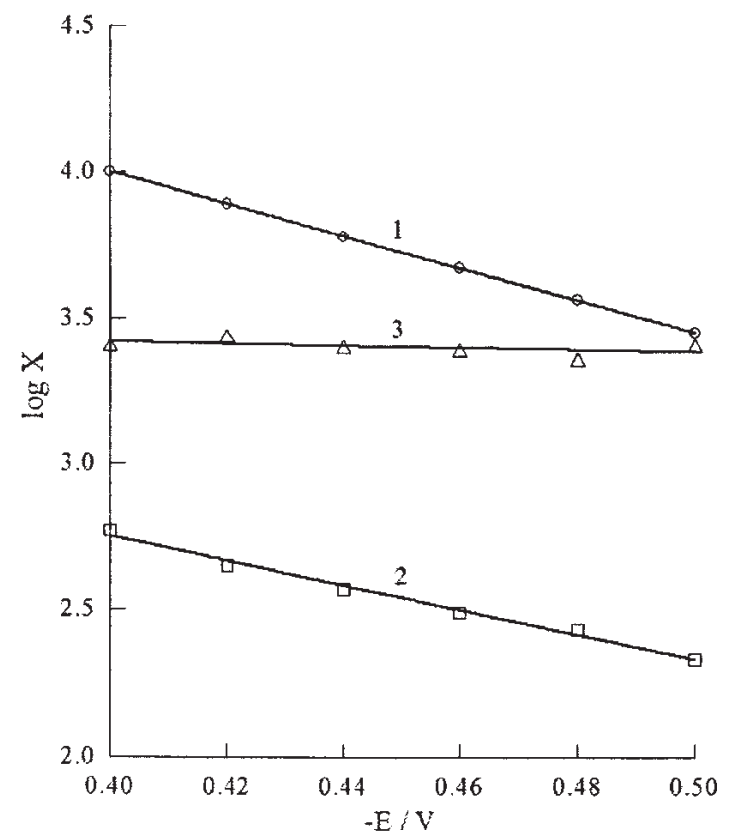

Рис. 8. Зависимости параметров эквивалентной схемы от потенциала $\mathrm{CoSi}_{2}$-электрода в $0.5 \mathrm{M} \mathrm{H}_{2} \mathrm{SO}_{4}, E_{f}=2.0 \mathrm{~B}$. $X=R_{1}(1), R_{2}(2), C_{2}$ (3). Сопротивления - в Ом·см², ем-

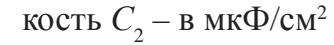

[Fig. 8. Potential dependences of equivalent electrical circuit parameters for $\mathrm{CoSi}_{2}$ electrode in $0.5 \mathrm{M} \mathrm{H}_{2} \mathrm{SO}_{4} E_{f}=2.0 \mathrm{~V}$. $X=R_{1}(1), R_{2}(2), C_{2}(3)$. The resistances are in $\Omega \mathrm{cm}^{2}$, the capacitance $C_{2}$ is in $\mu \mathrm{F} \mathrm{cm}^{-2}$ ] составляют $d \lg R_{1} / d E=5.5 \mathrm{~B}^{-1}, d \lg R_{2} / d E=4.0 \mathrm{~B}^{-1}$ и $d \lg C_{2} / d E \approx 0 \mathrm{~B}^{-1}$. Несмотря на отмеченное выше сходство формы графиков импеданса для анодированного и неанодированного $\mathrm{CoSi}_{2}$-электрода, характер зависимостей параметров эквивалентной схемы от $E$ для анодно окисленного электрода существенно отличается от зависимостей для электрода, не подвергавшегося окислению [29].

Результаты, представленные на рис. 8, не удается объяснить в рамках механизма разряд-электрохимическая десорбция, так как в этом случае либо $d \lg R_{2} / d E>d \lg R_{1} / d E$, либо $d \lg R_{2} / d E=d \lg R_{1} / d E$, но при этом уже $d \lg C_{2} / d E \neq 0$ [29]. Экспериментальные результаты более близки к теоретическим для механизма разряд-рекомбинация, в котором скорость-определяющей стадией является разряд ионов водорода, который в данном случае протекает с участием туннелирующих через оксидную пленку электронов. Для этого механизма, при ленгмюровской изотерме адсорбции водорода [29]:

$$
\begin{gathered}
R_{1}=\frac{R T}{\alpha_{1} F^{2}} \frac{1}{k_{1}} \\
R_{2}=\frac{R T}{\alpha_{1} F^{2}} \frac{1}{\sqrt{8 k_{1} k_{2}}} \\
C_{2}=\frac{\alpha_{1} q_{1} F}{R T} .
\end{gathered}
$$

Здесь $k_{1}$ и $k_{2}$ - константы скорости стадий разряда и рекомбинации соответственно, $\alpha_{1}-$ коэффициент переноса стадии разряда, $q_{1}$ - заряд, необходимый для образования монослоя адсорбированного водорода.

Теоретические значения наклонов, следующие из (5)-(7), составляют: $d \lg R_{1} / d E=\alpha_{1} F /(2.3 R T)$, $d \lg R_{2} / d E=\alpha_{1} F /(2 \cdot 2.3 R T), d \lg C_{2} / d E=0$, то есть наклон $\lg R_{2}, E$-зависимости должен быть в два раза меньше параметра $d \lg R_{1} / d E$, что не вполне соответствует опытным данным. Значение $\alpha_{1}$, найденное из наклона $\lg R_{1}, E$-зависимости, равно 0.32 , тогда как из наклона $\lg R_{2}, E$-зависимости -0.47 ; их среднее значение составляет 0.395 , что близко к $\alpha_{1}=0.42$, следующему из тафелевского наклона 0.138 В для данных условий (рис. 3). Экспериментальное значение $C_{2}=2530$ мкФ/см² (рис. 8) получается из (7) при $q_{1}=160$ мкКл/см², что является вполне правдоподобной величиной.

Можно предположить, что не вполне строгое соответствие теоретическим выражениям (5)-(7) связано с тем, что в них не введена вероятность туннелирования электронов, которая в общем случае зависит от потенциала электрода. Тем не менее выражение для плотности тока стадии разря- 
да $i_{1}$ можно записать в виде:

$i_{1}=F k_{1}^{0} P(E)(1-\theta) \exp \left(-\alpha_{1} F E / R T\right)=F k_{1} P(E)(1-\theta),(8)$ где $k_{1}^{0}-$ значение константы скорости стадии разряда при $E=0, \theta$-степень заполнения поверхности электрода атомами водорода, а для вероятности туннелирования, с учетом (4), использовать экспоненциальную зависимость:

$$
P=\exp \left[k_{P}\left(\Delta E_{b}-e_{0} \Delta E / 2\right)^{1 / 2}\right],
$$

где $\Delta E$ - поляризация, $e_{0}-$ заряд электрона, $k_{P}-$ константа. Тогда можно показать, что для механизма разряд-рекомбинация (при лимитирующей стадии разряда) наклон $\lg R_{2}, E$-зависимости также должен быть в два раза меньше, чем параметр $d \lg R_{1} / d E$. Одной из причин отсутствия количественного совпадения экспериментальных наклонов зависимостей $\lg R_{1}$ и $\lg R_{2}$ от $E$ с теоретически ожидаемыми может быть малое различие констант скоростей $k_{2}$ и $k_{1}$ в анализируемом интервале потенциалов.

Таким образом, несмотря на то, что импедансные данные не полностью соответствуют закономерностям простого механизма замедленный разряд-рекомбинация [29], согласие с этим механизмом можно считать лучшим среди проанализированных в [29] механизмов РВВ. Протекание РВВ на анодированном $\mathrm{CoSi}_{2}$ по механизму разряд-рекомбинация с лимитирующей стадией разряда означает: атомы водорода, образовавшиеся по реакции (2), слабо связаны с силанольными группами и достаточно легко вступают в реакцию рекомбинации с образованием $\mathrm{H}_{2}$.

\section{ВЫВОДЫ}

1. Обнаружено существенное различие в поведении анодных оксидных слоев на силицидах кобальта с низким $\left(\mathrm{Co}_{2} \mathrm{Si}\right)$ и высоким $\left(\mathrm{CoSi}_{2}\right)$ содержанием кремния при катодной поляризации в растворе серной кислоты. Оксидные пленки на $\mathrm{Co}_{2} \mathrm{Si}$ могут быть катодно восстановлены, тогда как пленки на $\mathrm{CoSi}_{2}$ не восстанавливаются при катодной поляризации в течение нескольких часов.

2. Вероятным механизмом выделения водорода на анодированном $\mathrm{CoSi}_{2}$-электроде в растворе $\mathrm{H}_{2} \mathrm{SO}_{4}$ является разряд протонов на границе оксидная пленка/раствор, протекающий при участии туннелирующих через оксидный слой электронов с последующей быстрой рекомбинацией атомов водорода.

3. Рост оксида на дисилициде кобальта в растворе серной кислоты не прекращается и в транспассивной области потенциалов.

\section{СПИСОК ЛИТЕРАТУРЫ}

1. Shamsul Huq A. K. M., Rosenberg A. J. // J. Electrochem. Soc., 1964, vol. 111, № 3, p. 270-278.

2. Tilak B. V., Ramamurthy A. C., Conway B. E. // Proc. Indian Acad. Sci. (Chem. Sci.), 1986, vol. 97, № 3-4, p. 359-393.

3. Vijh A. K., Bélanger G., Jacques R. // Materials Chemistry and Physics, 1989, vol. 21, p. 529-538.

4. Vijh A. K., Bélanger G., Jacques R. // Int. J. Hydrogen Energy, 1990, vol. 15, № 11, p. 789-794.

5. Vijh A. K., Bélanger G. // J. Mater. Sci. Lett., 1995, vol. 14, p. 982-984.

6. Поврозник В. С., Шеин А. Б. // Защчита металлов, 2007, т. 43, № 2, с. 216-221.

7. Kichigin V. I., Shein A. B. // Electrochim. Acta, 2015, vol. 164, p. 260-266.

8. Кичигин В. И., Шеин А. Б., Шамсутдинов А. Ш. // Конденсированные среды и межфазные границь, 2016, т. 18, № 3, с. 326-337.

9. Кичигин В. И., Шеин А. Б. // Вестник Тамбовского университета. Серия: Естественные и технические науки, 2013, т. 18, № 5, с. 2209-2212.

10. Шеин А. Б. Электрохимия силицицов и германидов переходных металлов. Пермь: Перм. гос. ун-т, 2009. 269 c.

11. Xu X., Bojkov H., Goodman D. W. // J. Vac. Sci. Technol., 1994, vol. A12, № 4, p. 1882-1885.

12. Кичигин В. И., Шеин А. Б. // Вестник Пермского ун-та. Химия, 2014, вып. 3(15), с. 4-13.

13. van Leeuwen H. P., Lyklema J. // Modern Aspects of Electrochemistry. No.17. New York, London: Plenum Press, 1986. P.411-483.

14. Vanheusden K., Warren W. L., Devine R. A. B. et al. // Nature, 1997, vol. 386, p. 587-589.

15. Zhang Q., Tang S., Wallace R. M. // Appl. Surf. Sci., 2001, vol. 172, № 1-2, p. 41-46.

16. Godet J., Pasquarello A. // Phys. Rev. Lett., 2006, vol. 97, № 15, p. 155901.

17. Küflüoglu H., Alam M. A. // IEEE Trans. Electron Devices, 2007, vol. 54, № 5, p. 1101-1107.

18. Кичигин В. И., Шеин А. Б. // Физикохимия поверхности и защчтта материалов, 2011, т. 47, № 2, c. $218-224$.

19. Weinberg Z. A., Rubloff G. W., Bassous E. // Phys. Rev. B, 1979, vol. 19, № 6, p. 3107-3117.

20. Ballarotto V. W., Breban M., Siegrist K., Phaneuf R. J., Williams E. D. // J. Vac. Sci. Technol., 2002, vol. B20, № 6, p. 2514-2518.

21. Gullikson E. M., Mills A. P., Phillips J. M. // Surface Science, 1988, vol. 195, № 1-2, p. L150-L154.

22. Ullah S. S., Robinson M., Hoey J., Driver M. S., Caruso A. N., Schulz D. L. // Semicond. Sci. Technol., 2012, vol. 27, № 6, p. 065012.

23. Wolters D. R., Zegers-Van Duijnhoven A. T. A. // Phil. Trans. Roy. Soc. London, 1996, vol. A354, p. 23272350. 
24. Zhao D., Zhu Y., Li R., Liu J.// Solid-State Electronics, 2005, vol. 49, № 12, p. 1974-1977.

25. Schmickler W., Schultze J. W. // Modern Aspects of Electrochemistry. No. 17. New York, London: Plenum Press, 1986. P. 357-410.

26. Schultze J. W., Vetter K. J. // Electrochim. Acta, 1973, vol. 18, № 11, p. 889-896.
27. Bao J., Macdonald D. D. // J. Electroanal. Chem., 2007, vol. 600, p. 205-216.

28. Попов Ю. А. Теория взаимодействия металлов и сплавов с коррозионно-активной средой. М.: Наука, 1995. $200 \mathrm{c}$.

29. Kichigin V. I., Shein A. B. // Electrochim. Acta, 2014, vol. 138, p. 325-333.

\title{
EFFECT OF ANODISING ON THE KINETICS OF THE HYDROGEN EVOLUTION REACTION ON COBALT SILICIDES IN SULPHURIC ACID SOLUTION
}

\author{
(C) 2017 V. I. Kichigin, A. B. Shein \\ Perm State University, 15 Bukirev str., 614990 Perm, Russia \\ e-mail: ashein@psu.ru; kichigin@psu.ru
}

Received 29.05.2017

\begin{abstract}
The effect of the anodizing of $\mathrm{Co}_{2} \mathrm{Si}$ and $\mathrm{CoSi}_{2}$ electrodes in $0.5 \mathrm{M} \mathrm{H}_{2} \mathrm{SO}_{4}$ with the potentials of oxide formation $E_{f}$ from 0.4 up to $2.0 \mathrm{~V}$ (standard hydrogen electrode) on the kinetics of hydrogen evolution reaction (HER) in $0.5 \mathrm{M} \mathrm{H}_{2} \mathrm{SO}_{4}$ at ambient temperature was studied. It has been discovered that the behavior of the anode oxide on cobalt silicides with low and high silicon content significantly differ: the oxide films on $\mathrm{Co}_{2} \mathrm{Si}$ obtained for all the $E_{\mathrm{f}}$ studied are cathodically reduced, but the oxide films on $\mathrm{CoSi}_{2}$ (close to $\mathrm{SiO}_{2}^{2}$ ) are retained in the cathodic region. Polarization curves were obtained for HER on $\mathrm{CoSi}_{2}$ with the thickness $d$ of the oxide film remaining practically unchanged during the measurements. The rate of HER on $\mathrm{CoSi}_{2}$ decreases by $\sim 2.5$ orders of magnitude with increasing $E_{f}$ from 0.5 to $2.0 \mathrm{~V}$. In the intervals $0.5 \leq E_{f} \leq 1.5 \mathrm{~V}$ and $1.8 \leq E_{f} \leq 2.0 \mathrm{~V}$ the current density of HER, with a constant cathodic potential, decreases exponentially with increasing $E_{f}$ (and, therefore, with increasing $d$ ), and in the $E_{f}$ region from 1.5 to $1.8 \mathrm{~V}$ there is a delay in the change of current density $i$. The linear dependence of lni on $E_{f}$ is explained in the framework of the mechanism of direct tunneling of electrons from the conduction band of cobalt disilicide to the unoccupied states of the redox system in the electrolyte. The delay in the change of the HER current at the potentials of formation of the oxide from 1.5 to $1.8 \mathrm{~V}$, which correspond to the beginning of the transpassive region for $\mathrm{CoSi}_{2}$, is explained by the development of an additional tunneling mechanism through the intermediate states, whose role can be played by the oxygen vacancies generated at the silicide/oxide interface upon transition from the passive to the transpassive state.

Based on impedance measurements on an anodized $\mathrm{CoSi}_{2}$ electrode in $0.5 \mathrm{M} \mathrm{H}_{2} \mathrm{SO}_{4}$, it was concluded that the cathodic hydrogen evolution at sufficiently low electrode potentials occurs via the dischargerecombination mechanism with the limiting discharge step (electron transfer to protonated silanol groups $\equiv \mathrm{Si}-\mathrm{OH}_{2}{ }^{+}$on the surface of the oxide film).
\end{abstract}

Keywords: cobalt silicide, anodising, hydrogen evolution reaction, electron tunneling.

\section{REFERENCES}

1. Shamsul Huq A. K. M., Rosenberg A. J. J. Electrochem. Soc., 1964, vol. 111, no. 3, pp. 270-278. DOI: $10.1149 / 1.2426107$

2. Tilak B. V., Ramamurthy A. C., Conway B. E. Proc. Indian Acad. Sci. (Chem. Sci.), 1986, vol. 97, no. 3-4, pp. 359-393. DOI: 10.1007/BF02849200

3. Vijh A. K., Bélanger G., Jacques R. Materials Chemistry and Physics, 1989, vol. 21, pp. 529-538. DOI: 10.1016/0254-0584(89)90151-X
4. Vijh A. K., Bélanger G., Jacques R. Int. J. Hydrogen Energy, 1990, vol. 15, no. 11, pp. 789-794. DOI: 10.1016/0360-3199(90)90014-P

5. Vijh A. K., Bélanger G. J. Mater. Sci. Lett., 1995, vol. 14, pp. 982-984. DOI: 10.1007/bf00274625

6. Povroznik V. S., Shein A. B. Protection of Metals, 2007, vol. 43, no. 2, pp. 203-207. DOI: 10.1134/ S0033173207020130

7. Kichigin V. I., Shein A. B. Electrochim. Acta, 2015, vol. 164, p. 260-266. DOI: 10.1016/j.electacta.2015.02.198 
8. Kichigin V. I., Shein A. B., Shamsutdinov A. Sh. Condensed Matter and Interfaces, 2016, vol. 18, no. 3, pp. 326-337. Available at: http://www.kcmf.vsu.ru/resources/t_18_3_2016_003.pdf

9. Kichigin V. I., Shein A. B. Bulletin of Tambov University: Natural and Technical Sciences, 2013, vol. 18, no. 5, pp. 2209-2212. Available at: http://cyberleninka.ru/article/ n/issledovanie-mehanizma-katodnyh-protsessov-na-silitsidah-kobalta-metodom-elektrohimicheskoy-impedansnoyspektroskopii.pdf

10. Shein A. B. Electrochemistry of Silicides and Germanides of Transition Metals. Perm, Perm State University, 2009. 269 pp.

11. Xu X., Bojkov H., Goodman D. W. J. Vac. Sci. Technol., 1994, vol. A12, no. 4, pp. 1882-1885. DOI: 10.1116/1.579022

12. Kichigin V. I., Shein A. B. Bulletin of Perm University: Chemistry, 2014, Issue 3(15), pp. 4-13. Available at: http://www.psu.ru/files/docs/ob-universitete/smi/nauchnyjzhurnal/khimiya/Him_2014_3.pdf

13. van Leeuwen H. P., Lyklema J. In: J. O’M. Bockris, B. E. Conway, R. E. White (Eds.), Modern Aspects of Electrochemistry. No. 17. New York, Plenum Press, 1986, pp. 411-483.

14. Vanheusden K., Warren W. L., Devine R. A. B. et al. Nature, 1997, vol. 386, pp. 587-589. DOI: 10.1038/ 386587a0

15. Zhang Q., Tang S., Wallace R. M. Appl. Surf. Sci., 2001, vol. 172, no. 1-2, pp. 41-46. DOI: 10.1016/S01694332(00)00839-4

16. Godet J., Pasquarello A. Phys. Rev. Lett., 2006, vol. 97, no. 15, p. 155901. DOI: 10.1103/PhysRevLett.97.155901

17. Küflüglu H., Alam M.A. IEEE Trans. Electron Devices, 2007, vol. 54, no. 5, pp. 1101-1107. DOI: 10.1109/ TED.2007.893809
18. Kichigin V. I., Shein A. B. Protection of Metals and Physical Chemistry of Surfaces, 2011, vol. 47, no. 2, pp. 272-276. DOI:10.1134/S2070205111020092

19. Weinberg Z. A., Rubloff G. W., Bassous E. Phys. Rev. B, 1979, vol. 19, no. 6, pp. 3107-3117. DOI: 10.1103/ PhysRevB.19.3107

20. Ballarotto V. W., Breban M., Siegrist K., Phaneuf R. J., Williams E. D. J. Vac. Sci. Technol., 2002, vol. B20, no. 6, pp. 2514-2518. DOI: 10.1116/1.1525007

21. Gullikson E. M., Mills A. P., Phillips J. M. Surface Science, 1988, vol. 195, no. 1-2, pp. L150-L154. DOI: 10.1016/0039-6028(88)90774-1

22. Ullah S. S., Robinson M., Hoey J., Driver M. S., Caruso A. N., Schulz D. L. Semicond. Sci. Technol., 2012, vol. 27, no. 6, pp. 065012. DOI: 10.1088/02681242/27/6/065012

23. Wolters D. R., Zegers-Van Duijnhoven A. T. A. Phil. Trans. Roy. Soc. London, 1996, vol. A354, pp. 2327-2350. DOI: $10.1098 /$ rsta.1996.0103

24. Zhao D., Zhu Y., Li R., Liu J. Solid-State Electronics, 2005, vol. 49, no. 12, pp. 1974-1977. DOI: 10.1016/j. sse.2005.09.010

25. Schmickler W., Schultze J. W. In: J. O’M. Bockris, B. E. Conway, R. E. White (Eds.), Modern Aspects of Electrochemistry. No.17. New York, Plenum Press, 1986, pp. 357-410.

26. Schultze J. W., Vetter K. J. Electrochim. Acta, 1973, vol. 18, no. 11, pp. 889-896. DOI: 10.1016/00134686(73)85043-1

27. Bao J., Macdonald D. D. J. Electroanal. Chem., 2007, vol. 600, pp. 205-216. DOI: 10.1016/j.jelechem. 2006.07.024

28. Popov Yu. A. Theory of Interaction of Metals and Alloys with Corrosive Medium, Nauka Publ., Moscow, 1995. 200 pp. (in Russian)

29. Kichigin V. I., Shein A. B. Electrochim. Acta, 2014, vol. 138, pp. 325-333. DOI: 10.1016/j.electacta.2014. 06.114
Кичигин Владимир Иванович - к. х. н., доцент кафедры физической химии химического факультета, Пермский государственный национальный исследовательский университет; тел.: +7(342) 2396452, e-mail: kichigin@psu.ru

Шеин Анатолий Борисович - Д. х. н., профессор, зав. кафедрой физической химии химического факультета, Пермский государственный национальный исследовательский университет; тел.: +7(342) 2396468, e-mail: ashein@psu.ru
Kichigin Vladimir I. - Dr. Sci. (Chem.), Associate Professor, Department of Physical Chemistry, Perm State University, ph.: +7(342) 2396452, e-mail: kichigin@psu.ru

Shein Anatoliy B. - Dr. Sci. (Chem.), Professor, Head of Physical Chemistry Department, Perm State University; ph.: +7(342) 2396468, e-mail: ashein@psu.ru 